\title{
Decreased expression of Dlg5 is associated with a poor prognosis and epithelial-mesenchymal transition in squamous cell lung cancer
}

\author{
Juanjuan Che ${ }^{1}$, Jing Wang ${ }^{1}$, Huihui Li $^{1}$, Hongchao Zhen ${ }^{1}$, Kun Shang ${ }^{1}$, Yan Yang ${ }^{2}$, Bangwei Cao ${ }^{1}$ \\ ${ }^{1}$ Department of Oncology, Beijing Friendship Hospital, Capital Medical University, Beijing, China; ${ }^{2}$ Department of Pathology, Beijing Friendship \\ Hospital, Capital Medical University, Beijing, China \\ Contributions: (I) Conception and design: B Cao, J Che; (II) Administrative support: B Cao; (III) Provision of study materials or patients: J Che, J \\ Wang, Y Yang; (IV) Collection and assembly of data: H Li, H Zhen; (V) Data analysis and interpretation: J Che, K Shang; (VI) Manuscript writing: \\ All authors; (VII) Final approval of manuscript: All authors. \\ Correspondence to: Bangwei Cao, PhD. Department of Oncology, Beijing Friendship Hospital, Capital Medical University, 95 Yong-an Road, Xi-cheng \\ District, Beijing 100050, China. Email: oncology@ccmu.edu.cn.
}

Background: Discs large homolog 5 (Dlg5) is a newly discovered member of the membrane-associated guanylate kinase superfamily (MAGUK) that is involved in several important processes, including the maintenance of epithelial cell polarity, cell proliferation control, and cell migration and invasion. Decreased expression of Dlg5 has been reported in malignancies arising from different organs. In the present study, we analyzed Dlg5 expression and its prognostic value in squamous cell lung cancer (SqCLC).

Methods: Tumor tissue and adjacent normal tissue sections were collected from 98 patients with SqCLC. The expression levels of Dlg5 and epithelial-mesenchymal transition (EMT) biomarkers in the tissue sections were examined by immunohistochemistry and western blot.

Results: There were 80 males and 18 females in the study cohort. Patients at pathological stages I and IIIA accounted for $64.3 \%$ and $35.7 \%$ of the cohort, respectively. Western blot showed that Dlg5 expression differed between SqCLC and healthy tissues. Western blot also revealed low Dlg5 expression to be associated with low E-cadherin expression and high vimentin expression, which was consistent with the findings of immunohistochemical staining. Dlg5 expression was significantly correlated with lymph node (LN) metastasis $(\mathrm{P}=0.001)$ and disease recurrence $(\mathrm{P}<0.001)$, as well as with E-cadherin and vimentin expression $(\mathrm{P}=0.025$ and $\mathrm{P}=0.001$, respectively). Univariate analysis showed that overall survival was significantly correlated with the tumor-node-metastasis $(\mathrm{P}<0.001)$ and $\mathrm{T}(\mathrm{P}=0.001)$ stages, LN metastasis $(\mathrm{P}<0.001)$, Dig5 expression $(\mathrm{P}<0.001)$, $\beta$-catenin expression $(\mathrm{P}=0.004)$, and vimentin expression $(\mathrm{P}=0.002)$. Patients with overexpression of $\mathrm{Dlg} 5$ and $\beta$-catenin had a more favorable prognosis than those without. Multivariate analysis revealed that tumor-node-metastasis stage [hazard ratio $(\mathrm{HR})=2.124 ; 95 \%$ confidence interval (CI), 1.195-3.777; $\mathrm{P}=0.010]$, Dlg5 expression ( $\mathrm{HR}=0.548 ; 95 \% \mathrm{CI}, 0.313-0.959 ; \mathrm{P}=0.035$ ), $\beta$-catenin expression $(\mathrm{HR}=0.545 ; 95 \% \mathrm{CI}, 0.312-0.953 ; \mathrm{P}=0.033)$, and vimentin expression $(\mathrm{HR}=1.850 ; 95 \% \mathrm{CI}, 1.050-3.258$; $\mathrm{P}=0.033$ ) could all independently predict the overall survival of patients with SqCLC.

Conclusions: Dlg5 is an important player in EMT which may have potential predictive value for SqCLC prognosis after surgery.

Keywords: Squamous cell lung carcinoma; squamous cell lung cancer (SqCLC); discs large homolog 5 (Dlg5); epithelial-mesenchymal transition (EMT); E-cadherin (E-cad)

Submitted Mar 10, 2021. Accepted for publication May 18, 2021.

doi: $10.21037 /$ jtd-21-752

View this article at: http://dx.doi.org/10.21037/jtd-21-752 


\section{Introduction}

Lung cancer (LC) ranks as the biggest contributor to tumor-associated death worldwide (1). Non-small cell lung cancer (NSCLC) comprises the bulk (85\%) of newly diagnosed LCs each year, and approximately $30 \%$ of these cases are further categorized as squamous cell carcinoma, which is also known as squamous cell LC (SqCLC).

SqCLC accounts for approximately 400,000 deaths worldwide every year (2). According to the World Health Organization, SqCLC can be classified into 4 subtypes: papillary, basal-like, clear cell, and small cell carcinoma. Although targeted therapies have led to significant advancements in the treatment of lung adenocarcinomas, little progress has been attained in the development of SqCLC-targeted therapies. SqCLC displays a high degree of malignancy, with hematogenous and lymphatic metastasis often occurring and $38 \%$ of patients displaying local or distant metastases during their initial consultation (3). Moreover, $22-55 \%$ of patients who receive a diagnosis of stage $1 \mathrm{SqCLC}$ develop metastases or experience disease recurrence after complete surgical resection of the primary tumor (4). The prognosis for patients with SqCLC is highly dependent on the occurrence of recurrence and metastasis. Therefore, the exploration of biomarkers involved in the invasion and metastasis of LC will provide insight that may aid in the development of new targeted therapies for SqCLC.

Discs large homolog 5 (Dlg5) is a newly discovered member of the membrane-associated guanylate kinase superfamily (MAGUK) (5). MAGUK proteins consist of 4 PDZ domains, 1 Src homology 3 (SH3) domain, and 1 guanylate kinase domain (6). Dlg5 is a multifunctional regulatory and scaffolding protein that interacts or binds to several proteins, including $\beta$-catenin, p55, kinesin family member 20A (KIF20A), syntaxin 4, and citron kinase (5,7-9). Dlg5 participates in a number of vital processes, such as the maintenance of epithelial cell polarity, cell proliferation control, and cell migration and invasion (9). Furthermore, Dlg5 stabilizes adherens junctions and tight junction complexes in brain, kidney, and lung tissues $(9,10)$. Recent studies have shown that $\mathrm{Dlg} 5$ is downregulated in many cancers, including those affecting the liver, prostate, pancreas, and bladder $(5,7,8,9)$. Dlg5 can also inhibit the migration of kidney epithelial cells and modify the epithelialmesenchymal transition (EMT) pattern in prostate cancer cells $(11,12)$. However, data regarding the value of Dlg5 in the prediction of LC prognosis are insufficient.

EMT is the process by which epithelial cells can convert into mesenchymal cells. EMT plays central roles in the invasion and metastasis of many cancers, including NSCLC (13-15). The mechanism of EMT involves the downregulation of classic adhesion molecules, including E-cadherin (E-cad) and $\beta$-catenin, as well as the upregulation of macrophage migration inhibitory factors, like vimentin. In a previous study, we found an association between decreased E-cad expression and an increased probability of metastatic spread to the lymph nodes (LNs) in SqCLC. Additionally, decreased E-cad and $\beta$-catenin expression were considered to be associated with an increased risk of disease recurrence and poor overall survival (16). Other studies demonstrated that the migration capacity of 2 SqCLC cell lines, H2170 and H1703, was significantly increased after the inhibition of E-cad and $\beta$-catenin $(14,15)$. Together, these findings attest to EMT being a critical player in SqCLC metastasis and invasion. Dlg 5 loss results in decreased expression of $\mathrm{N}$-cadherin, and the disintegration of the adherens junctions protein and loss of cell polarity. Decreased Dlg5 expression also causes the downregulation of atypical protein kinase $\mathrm{C}(\alpha \mathrm{PKC})$ in the apical domain of cells, leading to the misbalance of tight junctions (TJ) (10). The loss of epithelial cell polarity is a precursor to the development and metastasis of carcinomas in vivo, as it can lead to the failure of cell-cell adhesion and increases EMT in most cases.

In the present work, we examined Dlg5 expression in SqCLC tissues and adjacent normal healthy tissues, with the aim of identifying the effects and roles of $\mathrm{Dlg} 5$ in the invasion, metastasis, and prognosis of SqCLC. We firstly reported the role of $\operatorname{Dlg} 5$ in lung cancer, which is a newly discovered member of the membrane-associated guanylate kinase super family. And we proposed a novel Dlg5-EMT pathway in SqCLC which effect the invasion, metastasis, and prognosis of SqCLC. We present the following article in accordance with the REMARK reporting checklist (available at http://dx.doi.org/10.21037/jtd-21-752).

\section{Methods}

\section{Patients}

We retrospectively screened all individuals with SqCLC who received complete surgical resection of the primary tumor at Beijing Friendship Hospital from January 2004 to December 2012. The detailed enrollment process is shown in the flowchart in Figure 1. Patients were included in the study if they met the following conditions: (I) 


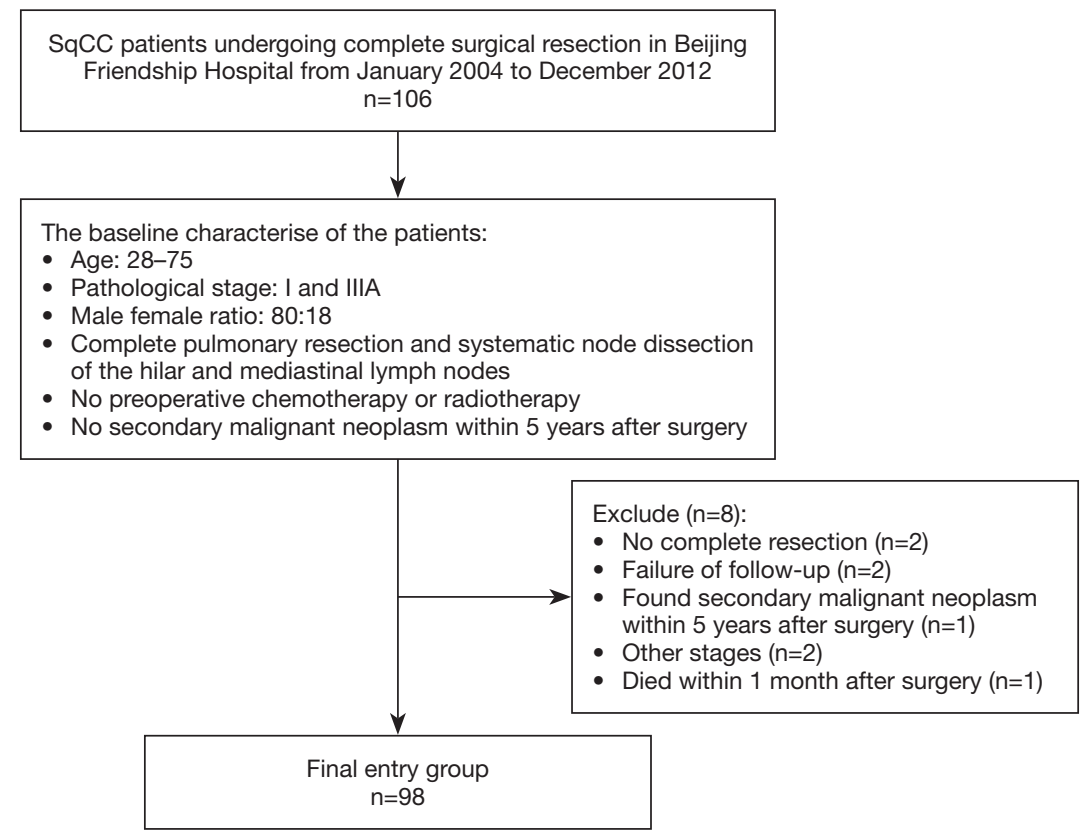

Figure 1 Flowchart. SqCC, squamous cell lung carcinoma.

had a diagnosis of SqCLC based on pathological tissue examination; (II) underwent complete resection of the LC and systemic hilar and mediastinal LNs; (III) had LC and normal tissues available for analysis; and (IV) were of Chinese ethnicity. The exclusion criteria were as follows: (I) patients who received preoperative chemotherapy or radiotherapy; (II) patients for whom tumor staging information was unavailable; (III) patients whose surgical resection was not carried out in accordance with the guidelines; (IV) patients with incomplete follow-up data or who died within 1 month after the operation; and (V) patients who were found to have a second primary malignant tumor within 5 years after the operation.

The Clinical Research Ethics Committee of the Beijing Friendship Hospital of Capital Medical University reviewed and issued approval for the current research. Each individual participant signed the written informed consent form prior to tissue sample testing. Each patient's tumor stage was re-evaluated based on the 7th edition American Joint Committee on Cancer (AJCC) LC guidelines (Table 1). The study conformed to the provisions of the Declaration of Helsinki (as revised in 2013).

\section{Immunobistochemistry}

Lung tissue samples from the participants were dewaxed using xylene and then rehydrated in ethanol at decreasing concentrations. Endogenous peroxidase activity was then blocked through 10-minute immersion of the tissues in 3\% hydrogen peroxide. Antigen recovery was done using 0.01 M sodium citrate buffer $(\mathrm{pH}=6.0)$, after which, the tissues were subjected to incubation in the presence of primary antibodies including: mouse anti-Dlg5 (1:150 dilution; Santa Cruz Biotechnology, CA, USA), mouse anti-E-cad (1:200 dilution; Cell Signaling Technology, Danvers, MA, USA), mouse anti- $\beta$-catenin (1:200 dilution; Cell Signaling Technology), and mouse anti-vimentin (1:400 dilution; Cell Signaling Technology). Following 3 washes with phosphatebuffered saline, the tissue samples were subjected to 30 minutes of incubation in the presence of the corresponding secondary antibodies at $37^{\circ} \mathrm{C}$, then 1 minute of incubation in the presence of 3,3'-diaminobenzidine (DAB). After counterstaining the tissue sections with hematoxylin, we used $85-100 \%$ ethanol to dehydrate them. Next, the slides were placed into xylene and the coverslips were applied with a mounting solution. Tissue sections known to express Dlg5, E-cad, vimentin, or $\beta$-catenin, served as a positive control.

The assessment of immunohistochemically (IHC) stained tissues was carried out by 2 pathologists independently. For the evaluation of membrane staining for Dlg5, E-cad, vimentin, and $\beta$-catenin, we employed the following semi- 
Table 1 Demographic and clinical characteristics of patients with squamous cell lung cancer

\begin{tabular}{|c|c|c|}
\hline Characteristic & No. & Percent (\%) \\
\hline \multicolumn{3}{|l|}{ Sex } \\
\hline Male & 80 & $81.6 \%$ \\
\hline Female & 18 & $18.4 \%$ \\
\hline \multicolumn{3}{|l|}{ Age (years) } \\
\hline$<65$ & 58 & $59.2 \%$ \\
\hline$\geq 65$ & 40 & $40.8 \%$ \\
\hline \multicolumn{3}{|l|}{ Smoking history } \\
\hline Smoker & 81 & $82.7 \%$ \\
\hline Never-smoker & 17 & $17.3 \%$ \\
\hline \multicolumn{3}{|l|}{ T stage } \\
\hline $\mathrm{T} 1$ & 26 & $26.6 \%$ \\
\hline $\mathrm{T} 2$ & 61 & $62.2 \%$ \\
\hline T3 & 11 & $11.2 \%$ \\
\hline \multicolumn{3}{|l|}{$\mathrm{N}$ stage } \\
\hline No & 63 & $64.3 \%$ \\
\hline $\mathrm{N} 1$ & 3 & $3.0 \%$ \\
\hline $\mathrm{N} 2$ & 32 & $32.7 \%$ \\
\hline \multicolumn{3}{|l|}{ TNM } \\
\hline Stage I & 63 & $64.3 \%$ \\
\hline Stage IIIA & 35 & $35.7 \%$ \\
\hline
\end{tabular}

quantitative scale: 0 points: an absence of color; 1 point: positive staining of $<25 \%$ of cells; 2 points: positive staining of $25-75 \%$ of cells; 3 points: positive staining of $>75 \%$ of cells. For simplification of the results, $0-1$ point was considered negative (low expression), while 2-3 points were considered positive (high expression).

\section{Western blot}

Radioimmunoprecipitation assay (RIPA) buffer was used for the extraction of total cellular and tissue proteins (BioRad, Hercules, CA, USA). To determine the protein concentrations of the samples individually, a Bradford Protein Assay (BioRad) was carried out. Next, we normalized the total protein concentrations, and $50 \mu \mathrm{g}$ of total protein was loaded into the wells of an $8-12 \%$ sodium dodecyl sulfatepolyacrylamide gel. We electrophoresized the proteins before transferring them to a polyvinylidene fluoride membrane (BioRad), which was subsequently incubated in the presence of primary mouse anti-human Dlg5 (1:1,000 dilution; Abcam, Cambridge, MA, USA), mouse anti-E-cad (1:500, Cell Signaling Technology), mouse anti-vimentin $(1: 1,000$, Cell Signaling Technology), and anti-tubulin (1:3,000, Santa Cruz Biotechnology, CA, USA) for $16 \mathrm{~h}$ at $4{ }^{\circ} \mathrm{C}$.

\section{Statistical analyses}

Continuous and categorical variables were expressed as means \pm standard deviations and percentages, respectively. We analyzed the associations of biological marker expressions with pathological parameters using $\chi^{2}$ tests. Cox proportional hazards regression was applied for univariate and multivariate analysis. The multivariate analysis solely included variables identified as being statistically significant in the univariate analysis, with the results presented as hazard ratios (HRs) with 95\% confidence intervals (CIs). Statistical differences in patients' overall survival were analyzed using the Kaplan-Meier method. $\mathrm{P}<0.05$ using 2-tailed tests was taken to show statistical significance. SPSS 18.0 (SPSS Inc., Chicago, IL, USA) was employed for all analyses.

\section{Results}

\section{Participant characteristics}

Table 1 details the characteristic information of the 98 patients with SqCLC at baseline. The study cohort comprised 80 males and 18 females, $40.8 \%$ of whom were aged $\geq 65$ years old. Of the study participants, $82.7 \%$ of the patients had smoking history. There were 26 (26.6\%), $61(62.2 \%)$, and $11(11.2 \%)$ cases of pathological $\mathrm{T}$ stages 1,2 , and 3 , respectively; and $63(64.3 \%), 3(3.0 \%)$, and $32(32.7 \%)$ cases of pathological $\mathrm{N}$ stages 0,1 , and 2 , respectively. The proportion of patients at pathological stage I and stage IIIA was $64.3 \%$ and $35.7 \%$, respectively.

\section{Dlg 5 expression is associated with the expression of EMT biomarkers}

Positive and negative staining for Dlg5 was detected in $57.1 \%$ and $42.9 \%$ of patient samples, respectively (Figure $2 A, B)$. Also, Positive and negative staining for E-cad and $\beta$-catenin were found to be expressed in the intercellular junctions (Figure 2C,D,E,F).

Membrane staining revealed that the levels of E-cad 

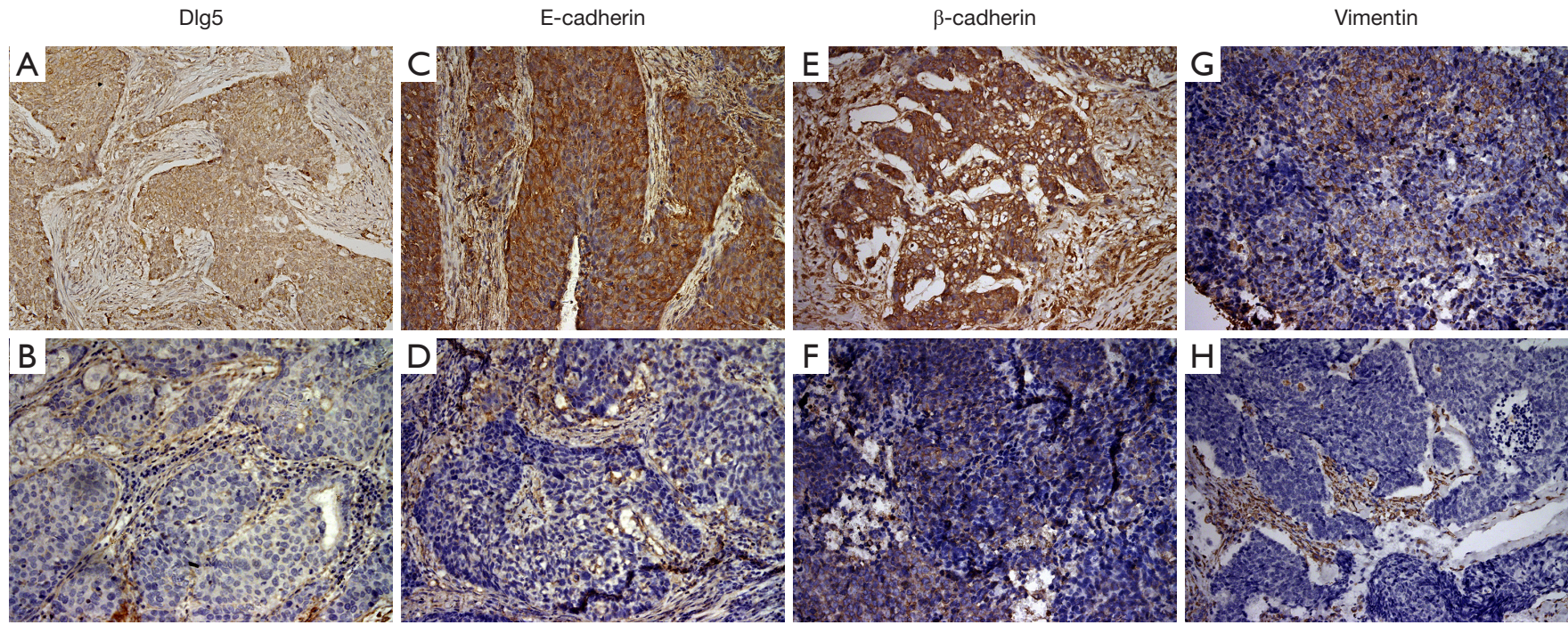

Figure 2 Expression of Dlg5, E-Cad, $\beta$-catenin, and vimentin in SqCLC samples, as detected by immunohistochemistry (IHC). (A,B) Dlg5, (C,D) E-cadherin, (E,F) $\beta$-catenin, and $(\mathrm{G}, \mathrm{H})$ vimentin expression were examined by IHC. A low expression of (B) Dlg5, (D) E-Cadherin, and (F) $\beta$-catenin, along with $(\mathrm{G})$ a high expression of vimentin, can be seen in the SqCLC tumor tissues (magnification $\times 200$ ). Dlg5, discs large homolog 5; E-Cad, E-cadherin; SqCLC, squamous cell lung cancer.

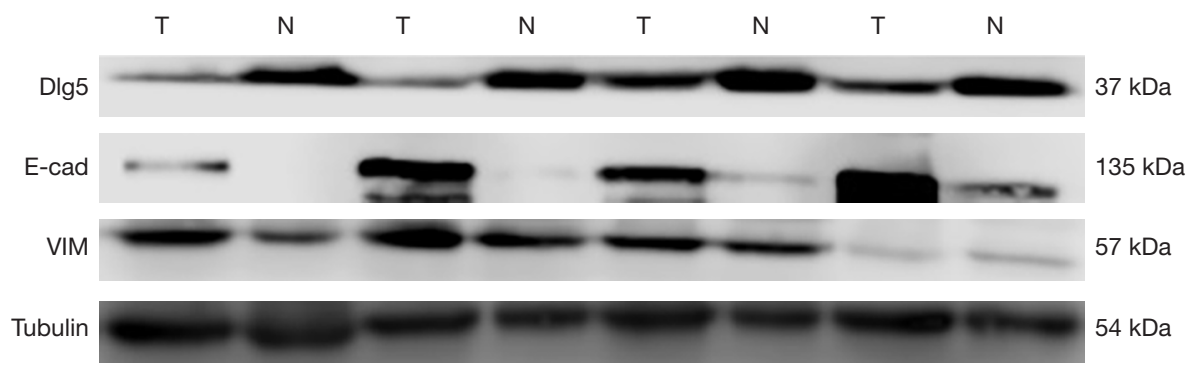

Figure $3 \mathrm{Dlg} 5$, E-cadherin, and vimentin expression levels were examined in SqCLC and adjacent healthy tissue samples from 4 patients. Total lysates were extracted from the tissues ( $\mathrm{N}$, adjacent normal tissue; $\mathrm{T}$, tumor tissue). For the western blot analysis, $\alpha$-Tubulin was used as a loading control. Dlg5 expression was lower in the SqCLC tissues than in the healthy tissues. Low Dlg5 expression was correlated with low E-cadherin and high vimentin levels. Dlg5, discs large homolog 5; E-Cad, E-cadherin; SqCLC, squamous cell lung cancer.

and $\beta$-catenin were decreased in many cancer cells (Figure 2D,F).

We detected low expression levels of E-cad and $\beta$-catenin in $53.1 \%$ and $58.2 \%$ of samples, respectively. Of the patient samples, $78.6 \%$ had low vimentin expression. Furthermore, the localization of vimentin in structures of the stroma was observed in both non-tumor and tumor tissue samples, with tumor tissues also displaying cytoplasmic vimentin localization (Figure 2G,H). To analyze the correlation between Dlg5 and EMT biomarkers, we first confirmed that Dlg5 expression differed between SqCLC and healthy tissues using western blot. The western blot analysis results uncovered an association between low Dlg5 levels and low E-cad levels in SqCLC tissues. However, SqCLC tissues displayed a high expression of vimentin. The western blot results were consistent with those obtained from IHC staining (Figure 3).

\section{The correlations between Dlg5 expression, EMT biomarker expression, and clinicopathologic parameters}

Dlg5 expression was found to be significantly associated with $\mathrm{LN}$ metastasis $(\mathrm{P}=0.001)$, tumor-node-metastasis stage $(\mathrm{P}=0.001)$, and recurrent disease $(\mathrm{P}<0.001)$ (Table 2). 
Table 2 Correlations of Dlg5, E-cadherin, $\beta$-catenin, and vimentin expression with clinicopathological parameters in SqCLC

\begin{tabular}{|c|c|c|c|c|c|c|c|c|c|c|c|c|}
\hline Variable & \multicolumn{3}{|c|}{ Dlg5 } & \multicolumn{3}{|c|}{ E-cadherin } & \multicolumn{3}{|c|}{$\beta$-catenin } & \multicolumn{3}{|c|}{ vimentin } \\
\hline Gender & & & 1.000 & & & 1.000 & & & 0.598 & & & 0.756 \\
\hline Male & 34 & 46 & & 42 & 38 & & 45 & 35 & & 62 & 18 & \\
\hline Female & 8 & 10 & & 10 & 8 & & 12 & 6 & & 15 & 3 & \\
\hline$<65$ & 29 & 29 & & 29 & 29 & & 29 & 29 & & 46 & 12 & \\
\hline$\geq 65$ & 13 & 27 & & 23 & 17 & & 28 & 12 & & 31 & 9 & \\
\hline Smoking history & & & & 0.423 & & & 0.605 & & & 0.788 & & \\
\hline Smoker & 33 & 48 & & 44 & 37 & & 48 & 33 & & 65 & 16 & \\
\hline Well & 4 & 2 & & 4 & 2 & & 4 & 2 & & 4 & 2 & \\
\hline Moderate & 28 & 52 & & 43 & 37 & & 47 & 33 & & 62 & 18 & \\
\hline Poor & 3 & 9 & & 7 & 5 & & 7 & 5 & & 10 & 2 & \\
\hline $\mathrm{T}$ & & & 0.17 & & & 0.262 & & & 0.164 & & & 0.828 \\
\hline $\mathrm{T} 1$ & 8 & 18 & & 15 & 11 & & 18 & 8 & & 20 & 6 & \\
\hline $\mathrm{T} 2$ & 27 & 34 & & 29 & 32 & & 31 & 30 & & 49 & 12 & \\
\hline T3 & 7 & 4 & & 8 & 3 & & 8 & 3 & & 8 & 3 & \\
\hline LN metastasis & & & $0.001^{*}$ & & & 0.673 & & & 0.527 & & & 0.210 \\
\hline Recurrence & & & $0.000^{*}$ & & & $0.001^{*}$ & & & $0.024^{*}$ & & & 0.629 \\
\hline No & 10 & 45 & & 22 & 35 & & 27 & 29 & & 45 & 11 & \\
\hline Yes & 32 & 11 & & 30 & 11 & & 30 & 12 & & 32 & 10 & \\
\hline
\end{tabular}

*, P values $<0.05$ were considered to be significant. SqCLC, squamous cell lung cancer; N0, no lymph node metastasis; N1-2, lymph node metastasis.

Among the patients with disease recurrence, $74.4 \%(32 / 43)$ expressed Dlg5 at a low level, with this rate dropping to $18.2 \%(10 / 55)$ for patients without recurrent disease $(\mathrm{P}<0.001)$. Furthermore, low $\mathrm{Dlg} 5$ expression was detected in $65.7 \%(23 / 35)$ of patients who eventually progressed to LN metastasis, but in only $30.2 \%(19 / 63)$ of patients without $\mathrm{LN}$ metastasis $(\mathrm{P}=0.001)$. Significant associations were also found between disease recurrence and a decreased expression of $\mathrm{E}$-cad $(\mathrm{P}<0.001)$ and $\beta$-catenin $(\mathrm{P}=0.024)$. However, vimentin expression was not significantly associated with clinicopathological parameters $(\mathrm{P}>0.05)$.

The correlations between the expression of Dlg5 and EMT biomarkers

Significant associations between Dlg5 expression and the 
Table 3 Correlations between Dlg5, E-cadherin, $\beta$-catenin, and vimentin expression

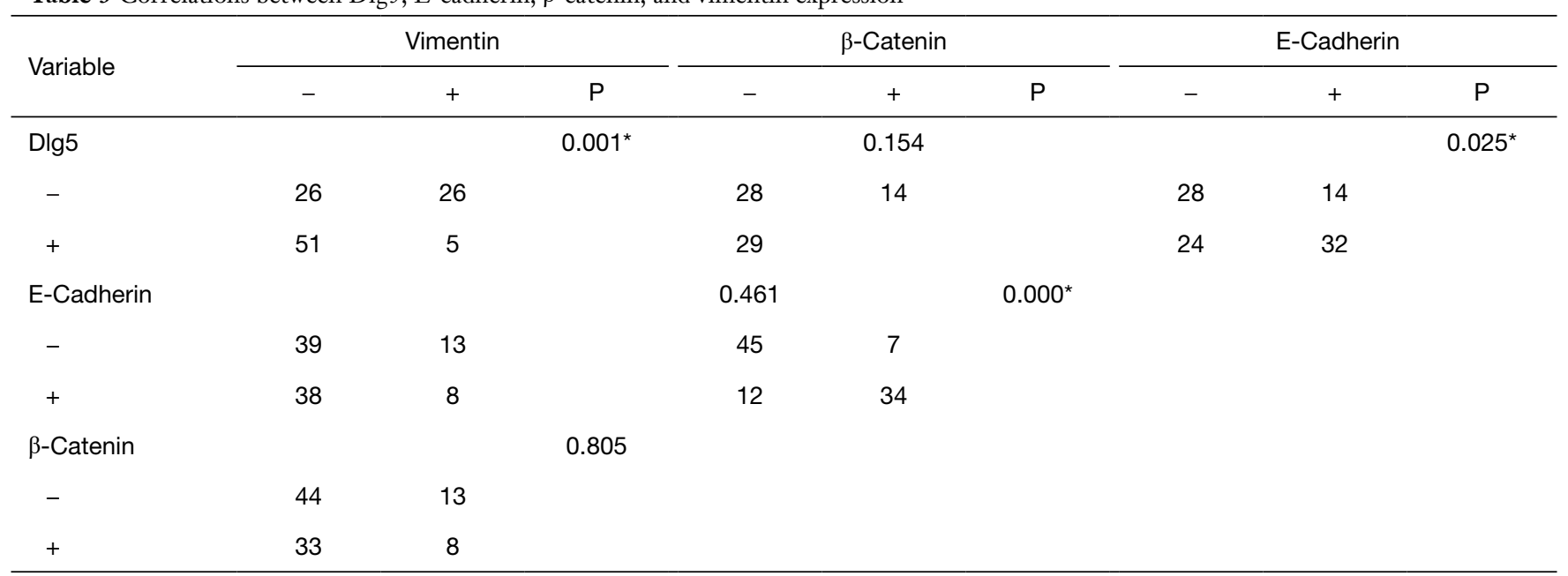

${ }^{*}$, P values $<0.05$ were considered to be significant.

expression of $\mathrm{E}$-cad and vimentin were uncovered $(\mathrm{P}=0.025$ and $\mathrm{P}=0.001$, respectively) (Table 3). The expression of E-cad also shared a close correlation with that of $\beta$-catenin $(\mathrm{P}<0.001)$ (Table 3). Meanwhile, vimentin expression was not significantly related to $\mathrm{E}$-cad $(\mathrm{P}=0.461)$ or $\beta$-catenin $(\mathrm{P}=0.805)$ expression (Table 3).

\section{TNM stage, Dlg5, vimentin, and $\beta$-catenin are independent predictors of OS in $S q C L C$}

At the final follow-up, 42 patients were alive and 56 patients had died. The univariate analysis (Table 4) revealed overall survival to have significant correlations with tumor-nodemetastasis stage $(\mathrm{P}<0.001$, Figure $4 A)$, $\mathrm{T}$ stage $(\mathrm{P}=0.001)$, and $\mathrm{LN}$ metastasis $(\mathrm{P}<0.001)$, as well as with $\mathrm{Dlg} 5(\mathrm{P}<0.001$, Figure $4 B), \beta$-catenin $(\mathrm{P}=0.004$, Figure $4 C)$, and vimentin $(\mathrm{P}=0.002$, Figure $4 D)$ expression. The prognosis of patients overexpressing $\operatorname{Dlg} 5$ and $\beta$-catenin was more favorable than that of patients expressing low levels of these 2 biomarkers. In contrast, patients expressing low levels of vimentin showed a better prognosis than patients with a high expression of vimentin.

The multivariate analysis revealed that tumor-nodemetastasis stage $(\mathrm{HR}=2.124 ; 95 \% \mathrm{CI}, 1.195-3.777$; $\mathrm{P}=0.010)$, Dlg5 expression (HR $=0.548$; 95\% CI, 0.313$0.959 ; \mathrm{P}=0.035), \beta$-catenin expression $(\mathrm{HR}=0.545 ; 95 \%$ CI, 0.312-0.953; $\mathrm{P}=0.033$ ), and vimentin expression $(\mathrm{HR}=1.850 ; 95 \% \mathrm{CI}, 1.050-3.258 ; \mathrm{P}=0.033)$ could all
Table 4 Univariate analysis of overall survival of patients with SqCLC

\begin{tabular}{lccc}
\hline Characteristic & No. & 5-year OS (\%) & P value \\
\hline Sex & & & 0.535 \\
Male & 80 & $46.4 \%$ & \\
Female & 18 & $48.9 \%$ & \\
Age (years) & & & 0.331 \\
$<65$ & 58 & $50.9 \%$ & \\
$\geq 65$ & 40 & $40.9 \%$ & \\
Smoking history & & & 0.235 \\
Smoker & 81 & $49.3 \%$ & \\
Never-smoker & 17 & $35.3 \%$ & \\
Tumor stage & & & \\
T1 & & & \\
T2 & 26 & $56.3 \%$ & $<0.001^{*}$ \\
T3 & 61 & $46.4 \%$ & \\
Lymph node stage & 11 & $0.00 \%$ & \\
N0 & & & \\
N1-2 & 63 & $62.7 \%$ & \\
TMN stage & 35 & $18.6 \%$ & \\
Stage I & & & \\
Stage IIIA & & & \\
\hline
\end{tabular}

Table 4 (continued) 
Table 4 (continued)

\begin{tabular}{|c|c|c|c|}
\hline Characteristic & No. & 5-year OS (\%) & $P$ value \\
\hline Chemotherapy (IIIA) & & & 0.766 \\
\hline Yes & 23 & $19.3 \%$ & \\
\hline No & 12 & $16.7 \%$ & \\
\hline Radiotherapy (IIIA) & & & 0.821 \\
\hline Yes & 11 & $22.7 \%$ & \\
\hline No & 24 & $16.7 \%$ & \\
\hline Dlg5 & & & $0.000^{*}$ \\
\hline+ & 56 & $61.2 \%$ & \\
\hline- & 42 & $23.8 \%$ & \\
\hline E-cadherin & & & 0.129 \\
\hline+ & 46 & $51.7 \%$ & \\
\hline- & 52 & $39.8 \%$ & \\
\hline$\beta$-catenin & & & $0.004^{\star}$ \\
\hline+ & 41 & $57.3 \%$ & \\
\hline- & 57 & $37.0 \%$ & \\
\hline Vimentin & & & $0.002^{*}$ \\
\hline+ & 21 & $28.6 \%$ & \\
\hline - & 77 & $50.1 \%$ & \\
\hline
\end{tabular}

$P$ values $<0.05$ were considered to be significant and are indicated with *. SqCLC, squamous cell lung cancer; NO, no lymph node metastasis; N1-2, lymph node metastasis; OS, overall survival; SqCC, squamous cell lung carcinoma.

independently predict the overall survival of patients with SqCLC (Table 5), which was in agreement with the observations of our previous study (16).

\section{Discussion}

In recent years, the constitution of non-small cell lung cancer has changed significantly. The incidence rate of adenocarcinoma has increased significantly, while squamous cell carcinoma has been decreasing year by year. In addition, according to the 2015 classification scheme of WHO, the subtyping of lung squamous cell carcinoma was modified to incorporate keratinizing, nonkeratinizing, and basaloid subtypes. It discontinued the terms of papillary carcinoma and small cell variant of squamous cell carcinoma. And clear cell change was regarded as one cytologic feature.

Metastasis is the chief reason for treatment failure in patients with cancer, making it 1 of the central challenges faced by the cancer research community. While the mechanisms involved in cancer metastasis are complex, exploration of these vital mechanisms will provide insight into new biological targets for therapeutic interventions. The present work explored the relevance of Dlg5 to SqCLC metastasis and prognosis. After detecting the expression of D $\lg 5$ in postoperative stage I and IIIA SqCLC tissues, we found that Dlg5 expression was significantly higher during the initial disease stages and decreased by the later disease stages. Associations between Dlg5 expression and $\mathrm{LN}$ metastasis and disease recurrence of SqCLC were also identified. As expected, Dlg5 expression was significantly higher in healthy tissues in comparison to SqCLC tissues, suggesting that Dlg5 plays a central role in maintaining healthy tissues. Further analysis revealed a relationship between the expression of Dlg5 and 2 EMT biomarkers, E-cad and vimentin, and survival analyses suggested that Dlg5 may be an independent factor influencing SqCLC prognosis.

The deregulated expression of Dlg5 may be associated with several factors, including disease stage, the occurrence of LN metastasis, and the disease recurrence rate. Decreased Dlg5 expression is also closely associated with shortened overall survival in patients with SqCLC, as evidenced by our Kaplan-Meier survival analysis. These findings are consistent with the results of other studies. For instance, Ke and colleagues (17) described the low level of Dlg5 detected in patients with hepatocellular carcinoma as being directly associated with advanced disease stage, as well as with reduced overall survival and disease-free survival rates. Furthermore, a low expression of D $\lg 5$ has been detected in prostate and bladder cancer, with similar reduced overall survival $(18,19)$. Moreover, patients with bladder cancer experiencing muscular invasion were reported to exhibit significantly lower Dlg5 levels than patients without muscular invasion. The results from the current study suggest that Dlg5 may serve as an excellent prognostic indicator in LC.

Dlg5 is a member of the MAGUK superfamily, and MAGUK family proteins are located at most cell-cell junctions. Previous reports have shown that Dlg5 may be associated with Crohn's disease (20), although the physiological functions of this connection remain unclear. Dlg5 may be a critical player in the regulation of cell migration, as well as the maintenance of cell polarity and adhesive junctions between cells $(9,10,21)$. Recent research has unveiled a relationship between abnormal Dlg5 

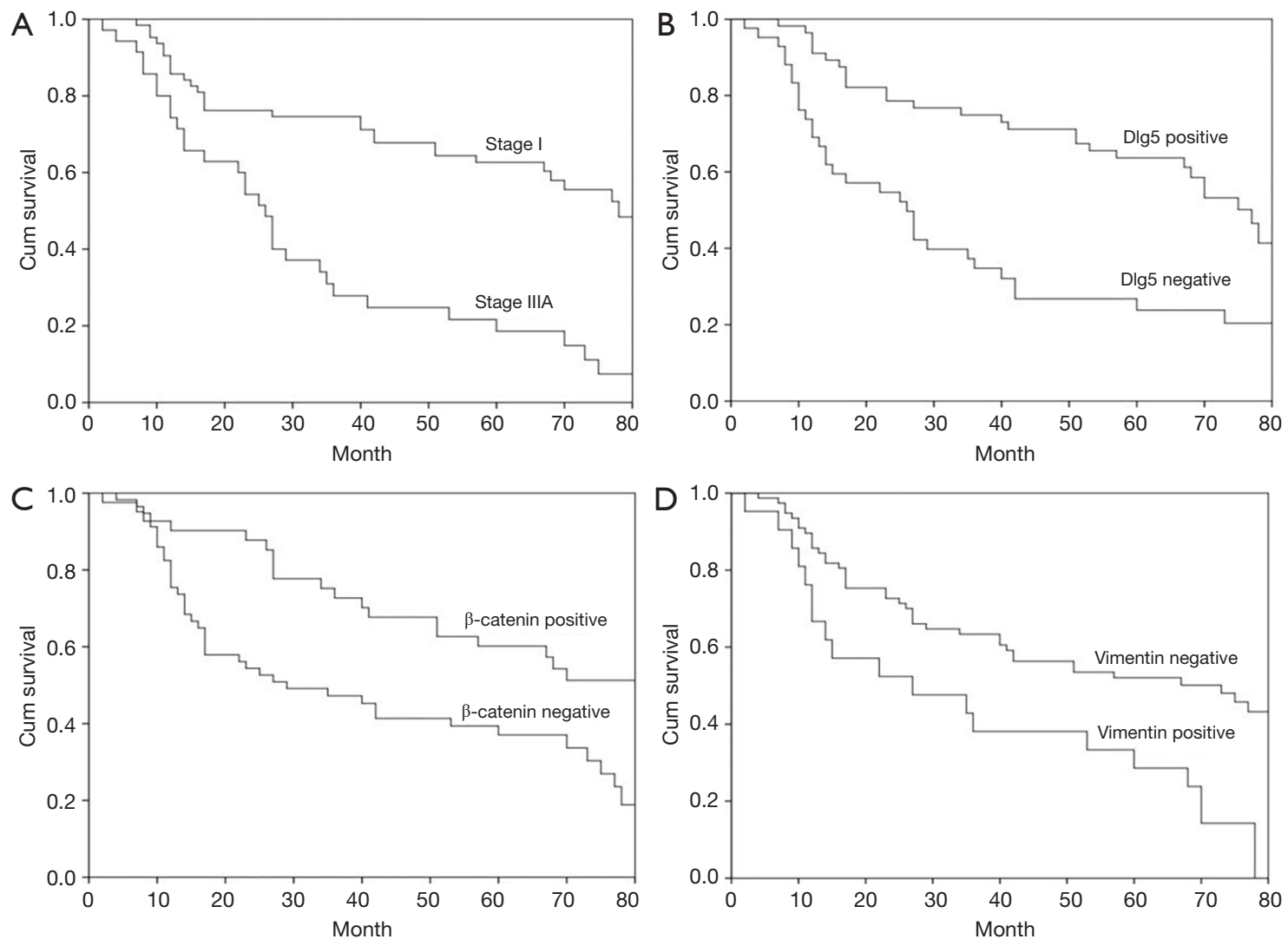

Figure 4 Survival curves of patients with SqCLC. (A) Cumulative survival curves of patients with stage I and IIIA SqCLC (log-rank test, $\mathrm{P}=0.010)$. (B) Cumulative survival curves of patients with Dlg5-positive and -negative tumors (log-rank test, $\mathrm{P}=0.035)$. $(\mathrm{C}) \mathrm{Cumulative}$ survival curves of patients with $\beta$-catenin-positive and -negative tumors (log-rank test, $\mathrm{P}=0.033$ ). (D) Cumulative survival curves of patients with vimentin-positive and -negative tumors (log-rank test, $\mathrm{P}=0.033$ ). Dlg5, discs large homolog 5; SqCLC, squamous cell lung cancer.

Table 5 Multivariate analysis of overall survival for SqCLC patients

\begin{tabular}{lccc}
\hline Variables & HR & $95 \% \mathrm{Cl}$ & P value \\
\hline TNM (I/IIA) & 2.124 & $1.195-3.777$ & $0.010^{*}$ \\
Dlg5 & 0.548 & $0.313-0.959$ & $0.035^{*}$ \\
$\beta$-Catenin & 0.545 & $0.312-0.953$ & $0.033^{*}$ \\
Vimentin & 1.850 & $1.050-3.258$ & $0.033^{*}$ \\
\hline
\end{tabular}

$P$ values $<0.05$ were considered to be significant and are indicated with *. SqCLC, squamous cell lung cancer; OS, overall survival; SqCC, squamous cell lung carcinoma; HR, hazard ratio; $\mathrm{Cl}$, confidence interval.

expression and tumor progression, with the downregulation of Dlg5 leading to enhanced tumor invasion and metastasis of prostate cancer through phosphorylation of Girdin (18). Additionally, the inhibition of Dlg5 in renal epithelial cells leads to the upregulation of several stromal markers, such as fibronectin and $\alpha$-smooth muscle actin, as well as the downregulation of epithelial markers, such as E-cad (22). Sezaki et al. demonstrated that Dlg5 could suppress EMT in renal epithelial cells by inhibiting transforming growth factor-beta signaling (12).

These findings suggest that Dlg5 may inhibit tumor cell invasion and metastasis by regulation of the factors involved in cell adhesion, cell polarity, and the EMT process. Therefore, we further investigated the correlation between Dlg5 and EMT biomarker proteins, and analyzed their relationship with the prognosis of SqCLC.

EMT is a critical biological process which sees malignant epithelial cells gain the ability to migrate and metastasize. EMT is associated with cancer invasion, metastasis, and a poor prognosis in most malignancies (23). There is a large bank of research which evidences EMT as being a pivotal step in NSCLC invasion and metastasis (13-15). For example, Zhou et al. analyzed the IHC staining of 312 
NSCLC tissues, which showed that the expression of E-cad and vimentin was directly correlated with an increased risk of NSCLC LN metastasis (13).

Our current research found that E-cad expression was decreased and vimentin expression was increased in SqCLC tissues. The IHC staining results showed that the expression levels of E-cad and vimentin were directly correlated with increased rates of $\mathrm{LN}$ metastasis and disease recurrence in patients with SqCLC. Univariate analysis revealed tumornode-metastasis stage, T-stage, LN metastasis, Dlg5 expression, $\beta$-catenin expression, and vimentin expression to all significantly predict overall survival in SqCLC. In the multivariate survival analysis, E-cad and vimentin were shown to be independent prognostic factors. As shown by western blot and IHC, Dlg5 had a positive correlation with E-cad but a negative correlation with vimentin. These findings suggest that Dlg5 can suppress SqCLC invasion and metastasis by inhibiting EMT.

In summary, $\mathrm{D} \lg 5$ is an independent prognostic factor for patients with SqCLC after surgical resection. Low Dlg5 expression is linked to advanced disease stages in patients with SqCLC. Moreover, decreased Dlg5 expression leads to the downregulation of E-cad and the upregulation of vimentin. This in-depth investigation of $\operatorname{Dlg} 5$ may provide insight into the mechanisms that contribute to the invasion and metastasis of SqCLC, helping researchers to identify new approaches for future targeted therapies for LC.

\section{Acknowledgments}

We would like to thank Mr. Yansong Huo for his assistance in the immunofluorescence staining experiments. We also would like to thank Mr. Liuwei Gao for his assistance with the western blotting experiments.

Funding: This study was supported by grants from the Research and Cultivation Fund of Capital Medical University (grant No. FYZ2017148) to Juanjuan Che, and the Beijing Natural Science Foundation (grant No. 7184200) to Lei Zhao.

\section{Footnote}

Reporting Checklist: The authors have completed the REMARK reporting checklist. Available at http://dx.doi. org/10.21037/jtd-21-752

Data Sharing Statement: Available at http://dx.doi. org/10.21037/jtd-21-752
Conflicts of Interest: All authors have completed the ICMJE uniform disclosure form (available at http://dx.doi. org/10.21037/jtd-21-752). The authors have no conflicts of interest to declare.

Ethical Statement: The authors are accountable for all aspects of the work in ensuring that questions related to the accuracy or integrity of any part of the work are appropriately investigated and resolved. Tissues were collected from the Beijing Friendship Hospital. The study protocol was approved by the Research Ethics Committees of Beijing Friendship Hospital. Written informed consent was obtained from all participants. The study conformed to the provisions of the Declaration of Helsinki (as revised in 2013).

Open Access Statement: This is an Open Access article distributed in accordance with the Creative Commons Attribution-NonCommercial-NoDerivs 4.0 International License (CC BY-NC-ND 4.0), which permits the noncommercial replication and distribution of the article with the strict proviso that no changes or edits are made and the original work is properly cited (including links to both the formal publication through the relevant DOI and the license). See: https://creativecommons.org/licenses/by-nc-nd/4.0/.

\section{References}

1. Isla D, Majem M, Viñolas N, et al. A consensus statement on the gender perspective in lung cancer. Clin Transl Oncol 2017;19:527-35.

2. Cancer Genome Atlas Research Network. Comprehensive genomic characterization of squamous cell lung cancers. Nature 2012;489:519-25.

3. Jemal A, Murray T, Ward E, et al. Cancer statistics. CA Cancer J Clin 2005;55:10-30.

4. Rami-Porta R, Ball D, Crowley J, et al. International Staging Committee; Cancer Research and Biostatistics; Observers to the Committee; Participating Institutions. The IASLC Lung Cancer Staging Project: Proposals for the revision of the $\mathrm{T}$ descriptors in the forthcoming (seventh) edition of the TNM classification for lung cancer. J Thorac Oncol 2007;2:593-602.

5. Nakamura H, Sudo T, Tsuiki H, et al. Identification of a novel human homolog of the Drosophila dlg, P-dlg, specifically expressed in the gland tissues and interacting with p55. Febs Letters 1998;433:63-7.

6. Liu J, Li J, Ren Y, et al. DLG5 in cell polarity maintenance 
and cancer development. Int J Biol Sci 2014;10:543-9.

7. Chang Y, Klezovitch O, Walikonis RS, et al. Discs large 5 is required for polarization of citron kinase in mitotic neural precursors. Cell Cycle 2010;9:1990-7.

8. Taniuchi K, Nakagawa H, Nakamura T, et al. Downregulation of RAB6KIFL/KIF20A, a kinesin involved with membrane trafficking of discs large homologue 5 , can attenuate growth of pancreatic cancer cell. Cancer Res 2005;65:105-12.

9. Nechiporuk T, Klezovitch O, Nguyen L, et al. Dlg5 maintains apical aPKC and regulates progenitor differentiation during lung morphogenes is. Dev Biol 2013;377:375-84.

10. Nechiporuk T, Fernandez TE, Vasioukhin V. Failure of epithelial tube maintenance causes hydrocephalus and renal cysts in dlg5(-1-) mice. Dev Cell 2007;13:338-50.

11. Sezaki T, Inada K, Sogabe T, et al. Role of Dlg5/lp-dlg, a membrane-associated guanylate kinase family protein, in epithelial-mesenchymal transition in LLc-PK1 renal epithelial cells. PLoS One 2012;7:e35519.

12. Sezaki T, Tomiyama L, Kimura Y, et al. Dlg5 interacts with the TGF-beta receptor and promotes its degradation. FEBS Lett 2013;587:1624-9.

13. Zhou L, Yu L, Wu S, et al. Clinicopathological significance of KAI1 expression and epithelial-mesenchymal transition in non-small celllung cancer. World J Surg Oncol 2015;13:234.

14. Yue D, Li H, Che J, et al. EMX2 is a predictive marker for adjuvant chemotherapy in lung squamous cell carcinomas. PLoS One 2015;10:e0132134.

15. Yue $\mathrm{D}, \mathrm{Li} \mathrm{H}$, Che J, et al. Hedgehog/Gli promotes epithelial-mesenchymal transition in lung squamous cell

Cite this article as: Che J, Wang J, Li H, Zhen H, Shang K, Yang Y, Cao B. Decreased expression of Dlg5 is associated with a poor prognosis and epithelial-mesenchymal transition in squamous cell lung cancer. J Thorac Dis 2021;13(5):3115-3125. doi: $10.21037 /$ jtd-21-752 carcinomas. J Exp Clin Cancer Res 2014;24;33:34.

16. Che J, Yang Y, Xiao J, et al. Decreased expression of claudin-3 is associated with a poor prognosis and EMT in completely resected squamous cell lung carcinoma. Tumour Biol 2015;36:6559-68.

17. Ke Y, Bao T, Zhou Q, et al. Discs large homolog 5 decreases formation and function invadopodia in human hepatocellular carcinoma via Girdin and Tks5. Int J Cancer 2017;141:364-76.

18. Tomiyama L, Sezaki T, Matsuo M, et al. Loss of Dig5 expression promotes the migration and invasion of prostate cancer cells via Girdinphosphorylation. Oncogene 2015;34:1141-9.

19. Zhou Z, Guo Y, Liu Y, et al. Methylation-mediated silencing of Dlg5 facilitates bladder cancer metastasis. Exp Cell Res 2015;331:399-407.

20. Shafieyoun A, Moraveji S, Bashashati M, et al. Discs Large Homolog 5 (DLG5) Gene Polymorphism and Crohn's Disease: A Meta-Analysis of the Published Studies. Acta Med Iran 2016;54:289-95.

21. Smolen GA, Zhang J, Zubrowski MJ, et al. A genomewide RNAi screen identifies multiple RSK-dependent regulators of cell migration.Genes Dev 2010;24:2654-65.

22. Sezaki T, Inada K, Sogabe T, et al. Role of Dlg5/lp-dlg, a membrane-associated guanylate kinase family protein, in epithelial-mesenchymal transition in LLc-PK1 renal epithelial cells. PLoS One 2012;7:e35519.

23. Santamaria PG, Moreno-Bueno G, Portillo F, et al. EMT: Present and future in clinical oncology. Mol Oncol 2017;11:718-38.

(English Language Editor: J. Reynolds) 